\title{
Movement is governed by rotational population dynamics in spinal motor networks
}

\author{
Henrik Lindén, ${ }^{1 *}$ P. C. Petersen, ${ }^{2}$, M. Vestergaard ${ }^{3}$, Rune W. Berg ${ }^{1 *}$ \\ ${ }^{1}$ Department of Neuroscience, Faculty of Health and Medical Sciences, University of Copenhagen \\ Blegdamsvej 3, 2200 Copenhagen, Denmark \\ 2 Neuroscience Institute, NYU Langone \\ New York University, New York, NY 10016, USA \\ ${ }^{3}$ Department of Neuroscience, Max-Delbrück Center for Molecular Medicine \\ in the Helmholtz Association (MDC), Berlin-Buch \\ Robert-Rössle-Strasse 10, 13092 Berlin, Germany \\ *Correspondence; E-mail: runeb@sund.ku.dk (RWB), hlinden@sund.ku.dk (HL).
}

\section{ABSTRACT}

Although the nervous system is elegantly orchestrating movements, the underlying neural principles remain unclear. Since flexor- and extensor-muscles alternate during movements like walking, it is often assumed that the responsible neural circuitry is similarly alternating in opposition. Here, we present ensemble-recordings of neurons in the lumbar spinal cord that indicate that, rather than alternation, the population is performing a "rotation" in neural space, i.e. the neural activity is cycling through all phases continuously during the rhythmic behavior. The radius of rotation correlates with the intended muscle force. Since existing models of spinal motor control offer an inadequate explanation of rotation, we propose a new theory of neural generation of movement from which this and other unresolved issues, such as speed regulation, force control, and multi-functionalism, are conveniently explained.

The neural circuitry behind movement encompasses several distinct forebrain regions, cerebellum and the brainstem. The core executive circuits for movement such as locomotion, however, reside in the spinal cord ${ }^{1}$. These spinal motor circuits, often referred to as central pattern generators (CPGs), are capable of autonomous generation of rhythmic coordination of muscles. Although great progress has been made in characterizing the cellular properties of spinal inter- and motor neurons, including their genetic lineages ${ }^{2,3}$, the network architecture as well as the associated neuronal ensemble dynamics remain elusive. Due to the apparent right-left and flexor-extensor alternation, it has often been proposed that distinct groups of interneurons, or 'modules', are active in a push-pull fashion and that the rhythm is ensured by cellular pacemaker properties ${ }^{4,5}$. It is unknown if and how such organization and different motor programs are manifested in ensemble activity of spinal networks.

\section{Rotation in spinal motor circuits}

Here, we examined the activity in spinal motor networks using extracellular multi-electrode recording in the turtle lumbar spinal cord. This preparation provided mechanical stability, which allowed simultaneous monitoring of large numbers of spinal interneurons in laminae VII-VIII and motoneurons during the execution of various rhythmic motor programs ${ }^{6-8}$. As expected, the activity of individual neurons was rhythmic in relation to the nerves, but the population activity as a whole appeared rather incomprehensible (Fig. 1a-b). However, when sorting these neurons according to phase of the motor nerve output we found that the population activity resembled a continuous sequence, that covered all phases of the cycle (Fig. 1c). To better understand the sequential activity, we performed a principal component analysis (PCA) of both the neuronal population and the nerve activity. Both the neuronal activity and 6 motor nerves followed a low-dimensional manifold, i.e. most variance was explained by few components (Fig. 1d). Whereas the nerve activity appeared entangled, the neuronal activity had a simple rotation (Fig. 1e-f). Rotational population activity was independent on the sorting and it was observed in all trials and across animals (Extended data Fig. 1-2, Supplementary Video 1). These data indicate that, rather than alternation, the neuronal population is executing a "rotation", i.e. the ensemble is cycling through all phases. There did not seem to be any discrete phase preference as otherwise expected in an alternating modular network (Extended data Fig. 1-2). Rotational dynamics has been observed in the motor cortex and elsewhere ${ }^{9-11}$, but it has not been described for spinal circuits previously. Nevertheless, indications can be found as wide phase-distributions in the scarce literature on spinal population recordings ${ }^{6,12-14}$. 

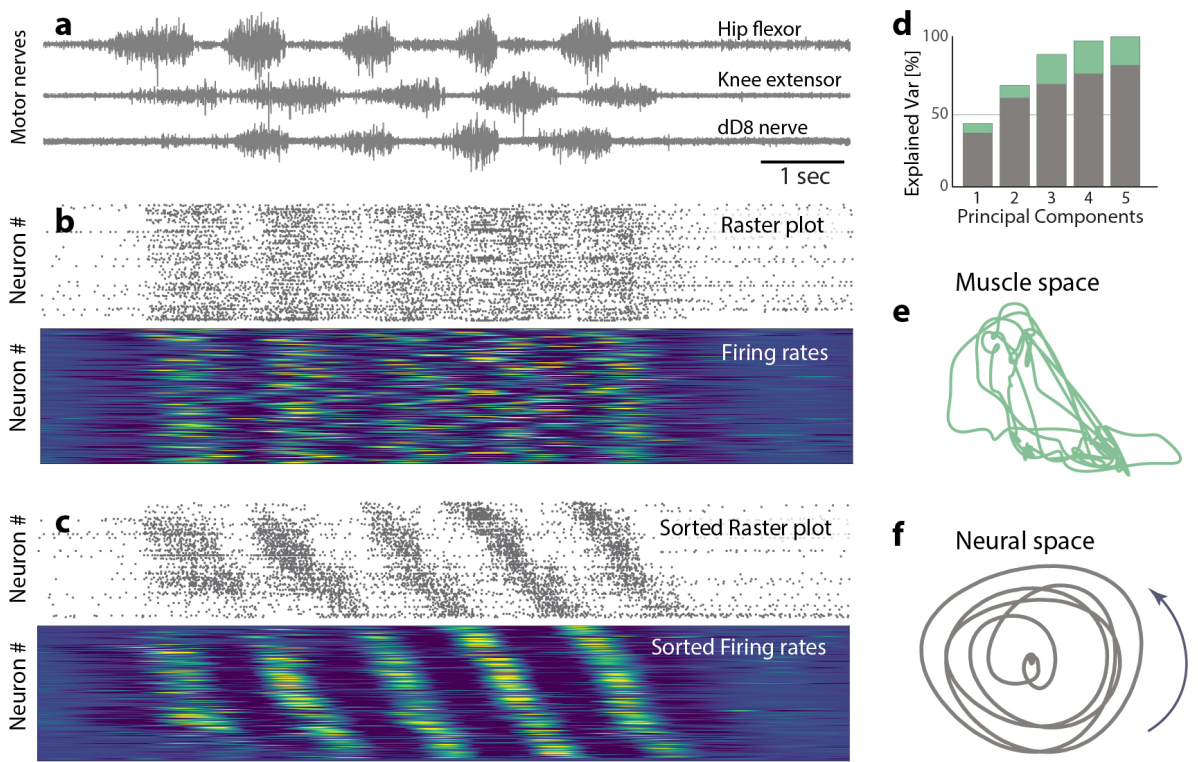

Fig. 1. Neuronal population activity in the lumbar spinal cord has rotational dynamics. a, Activity of 3 selected motor nerves (ENG) during rhythmic hindlimb movement. b, Concurrent ensemble activity in the turtle lumbar spinal cord as raster of spinal neurons (top, $\mathrm{n}=214$ ) and estimated firing rates (bottom). c, Sorting the neurons in (b) according to phase (hip flexor) reveals sequential activity. d, First principal components explains most variance of ENG activity (green) and neuronal ensemble activity (gray). First two principal components of nerve activity, e, and neuronal population, $\mathbf{f}$.

\section{New theory to explain rotation and central pattern generation}

Because conventional CPG models, that are founded on a push-pull organization with intrinsically rhythmic modules ${ }^{15,16}$, do not readily explain rotational dynamics, we sought to conceive a new theory that can account for this and other open questions in motor control. In particular, the mechanisms for generation of rhythms have remained nebulous. Cellular pacemaker properties has been suggested ${ }^{4}$, but decades of research has not been able to pinpoint a responsible cell type ${ }^{16}$. Here, we propose that the rhythm arises from the network rather than via cellular properties. We show that a network, which is close to the transition point of dynamical instability, can have rhythmogenetic properties without requiring specific cellular properties. Since the CPG network structure is unknown, we parsimoniously assumed a structure, where glutamatergic neurons were randomly and recurrently connected. To prevent catastrophic run-away activity ${ }^{17,18}$ the excitation (E) was balanced by recurrent glycinergic inhibition (I) (Fig. 2a-b), in line with reports of balanced synaptic input in various motor circuits ${ }^{19-21}$. Balanced networks of this type are known to undergo a phase transition when synaptic weights are increased beyond a critical value ${ }^{22,23}$. For large networks, activity in this regime is chaotic ${ }^{24}$, whereas finite-sized networks in a dynamical regime close to the transition point may display more regular activity ${ }^{25}$. A linearization of the dynamics close to this point (Supplementary information) demonstrated that finitely-sized networks can generate oscillatory activity if the leading eigenvalue of the connectivity matrix has a non-zero imaginary part ${ }^{25}$. Based on this idea, we set up a model network of rate-neurons with sparse connectivity where an input drive, e.g. sensory-related or descending from the brain, could move the eigenvalues of the connectivity matrix across the stability line due to change in set-point of the firing-rate function (Fig. 2c-d). As the input was increased beyond a critical level (red dashed line) the firing rates in the network displayed self-sustained rhythmic activity (Fig $2 \mathbf{e}$ ). Unexpectedly, when sorting the neurons according to phase, a sequential activity was revealed, i.e. a rotation, similar to the experimental observations (Fig. 2f-g). We dub the model the "Balanced Sequence Generator" (BSG). Both the BSG-model and the experimentally observed rotation are fundamentally different from conventional models where the neurons have clustered phase preferences and modules composed exclusively of excitatory neurons.

\section{Comparison between theory and experiment}

To model the output nerve activity from the BSG-network we connected a subset of neurons based on their phase in the dominant eigenmode to pools of motor neurons to provide the appropriate nerve activity. This resulted in an alternating nerve output in resemblance with experiments (Fig. 2h-i, Supplementary Video 2). Next, we investigated the behaviors of the excitatory and inhibitory populations during the motor program in the BSG-model. It turns out that both the E- and I-populations themselves undergo rotation (Extended data Fig. 3). These results demonstrate that rotational dynamics can arise in remarkably simple networks without fine-tuning of parameters and result in an alternating nerve output, in line with our experimental findings 

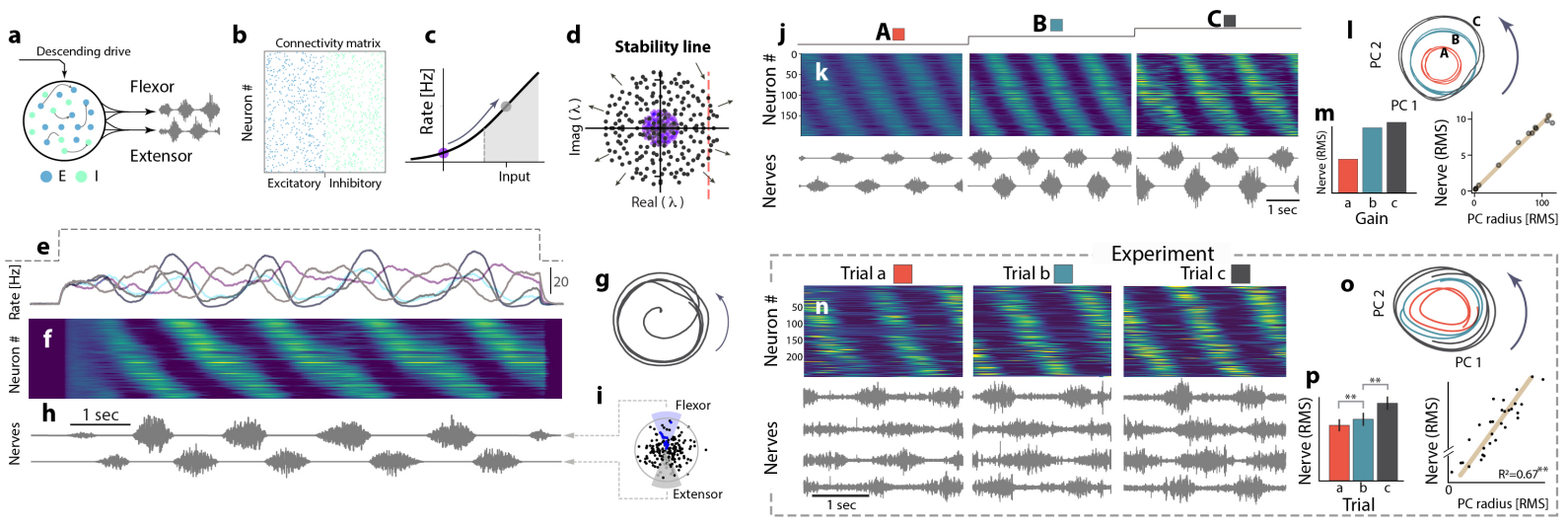

Fig. 2. Rotational dynamics emerges in the BSG-model. a, Sketch of the BSG-model: An input drive activates a recurrent network with excitatory (blue) and inhibitory (green) neurons. A subset of cells provides motor output. b, Connectivity matrix has 50\% excitation and inhibition. c, The firing rate is increased by a common drive (arrow) causing the eigenvalue spectrum to expand (purple vs. gray, $\mathbf{d}$ ) and cross the stability line (broken red line) and thus generate a network oscillation. e, Input (top) and firing rates of 5 neurons (bottom). f, Sequential activity revealed by sorting according to phase, similar to experiments. $\mathbf{g}$, The ensemble PCs exhibit rotation. $\mathbf{h}$, Flexor/extensors innervated by anti-phase neurons in the strongest eigenmode (blue/gray), i. j, Increasing drive (A-C) ramps up ensemble activity, $\mathbf{k}$, and nerve output (bottom). l, Higher firing rates are associated with larger radius of rotation in PC-space. $\mathbf{m}$, Correlation between radius, drive and nerve output. $\mathbf{n}$, experimental verification: Trials a, b and $\mathrm{c}$ with different nerve output and radius (o, color-matched). p, nerve amplitude (RMS $\pm \mathrm{SE}$ of 6 nerves) vs. radius of rotation (RMS of PC1 and 2). o, Wilcoxon test, Linear regression, $* *: p<0.01$, F-statistics.

(Supplementary Video 2). Although proprioceptive feedback from muscles and their reflexive circuitry were not included in the BSG-model, these are expected to improve the performance.

\section{Control of force and period}

Next, we evaluated whether the BSG-model could embrace previously unsolved issues, such as independent control of force and speed of the rhythm. The ability to modulate the strength of the output and speed is key for volitional control, but no mechanisms has been proposed for controlling these independently to our knowledge. First, we found that collective modulation of the gain by an input drive could indeed control the amplitude in the BSG-model (Fig. 2j-k). As the amplitude increased so did the radius of rotation, while the frequency and sequence remained largely unaltered (Fig. 21-m, Extended data fig. 4). To verify this prediction experimentally we inspected trials that, due to an inherent variability, had various radii of rotation (Fig. 2n, Extended data figure 1c). The radius of rotation had substantial correlation with the motor nerve activity (Fig. 2o, Extended data figure 5), which provide support for the BSG-model and the mechanism of muscular control.

Next, we explored the capacity of the BSG-model to control the period of the rhythm. Rather than collectively adjusting the gain, modulation of the frequency without affecting the amplitude was indeed possible by selective gain-modulation (Fig. 3). Individual gain-modulation is a powerful tool in network control $^{26}$ and here we systematically tuned the neuronal gain to identify a subset of neurons that had most influence on the period. A systematic perturbation of gain revealed an uneven influence on the rhythm (Fig. 3a-f). Some neurons had a strong either positive or negative effect, which we call "brake-" and "speed cells", respectively, while others had small effects on the rhythm. There were both inhibitory and excitatory neurons among both the speed- and the brake cells (Fig. 3g-h). Interestingly, cells with a speed-modulating capacity have been demonstrated experimentally ${ }^{27,28}$. Since both excitatory and inhibitory members were found among the brake- and speed cell categories in our model, we propose this as an experimental prediction. The modulation capacity is not due to cellular properties, but rather their location in the network. A possible link between the network location, cell identity, and speed control remains to be assessed.

\section{Population activity of multiple motor programs}

The ability to execute multiple motor outputs, i.e. a multifunctional output, is the hallmark of the motor system ${ }^{29}$. Contriving a network model that can accommodate the rich repertoire of motor behaviors has so far been a major challenge. For this reason, we explored the potential of generating multiple motor patterns by selective activation within the BSG-network. We used two well-known motor behaviors in turtles and investigated these both experimentally and in the model. These behaviors consist of hind limb movements, where either the knee is protracted while moving the foot in small circles (pocket scratching) or protracting the foot with stretched leg (rostral scratching $)^{30}$. Indeed, the BSG-model was able to produce qualitatively 

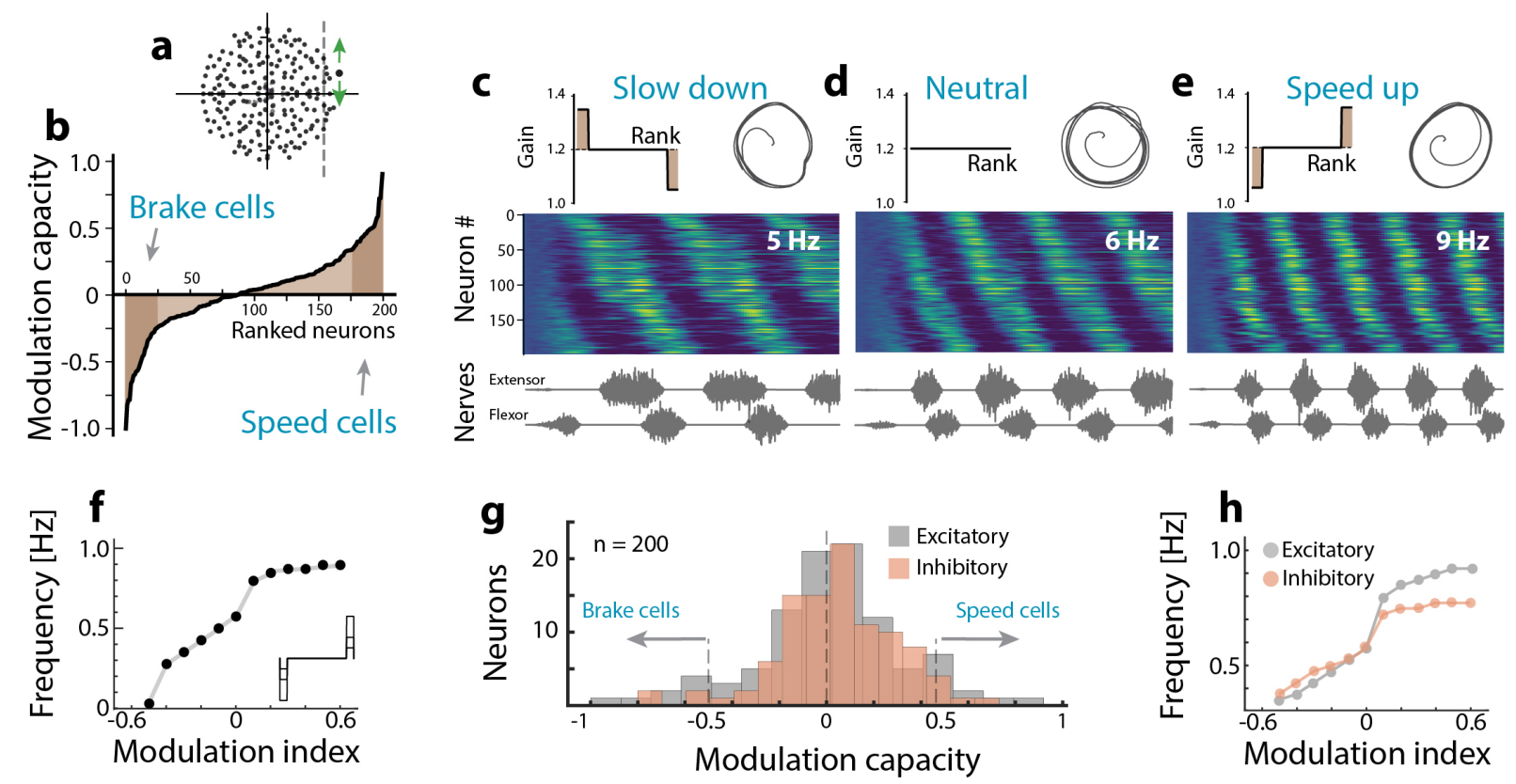

Fig. 3. Modulation of period in the BSG-model. a, Adjusting the drive to a neuron changes the rhythm by moving the eigenvalue up or down (green arrows). b. Capacity to modulate the rhythm by an individual neuron is assessed by changing drive (gain). Ranking neurons accordingly reveals "brake-" and "speed cells". c, When activating brake cells while impeding speed cells (activation profile, top left), the rhythm is slowed down compared with neutral, d. Sorted ensemble activity (middle) and nerve output (bottom). Radius of rotation is largely unchanged (PCs top right) indicating a similar amplitude of motor output. e, Reversed activation results in faster rhythm $(9 \mathrm{~Hz})$. f, Gradually modulating the speed/brake cells (inset) can either decrease or increase frequency. $\mathbf{g}$ Capacity to modulate the rhythm has a bell-shaped distribution. Brake and speed cells represent cells with strong modulation capacity, in which, both excitatory and inhibitory cells are found. h, Modulating only excitatory (gray) or inhibitory (orange) cells is sufficient to modulate the frequency. 
similar patterns. This was accomplished by identifying two distinct neuronal input sets for gain-modulation in the network (Fig. 4a-b, Supplementary Video 3). A small group of output neurons drive the motor neurons and the consequent movement was induced (Fig. 4c). The output of behavior 1 had knee/hip extensors in phase ('no shift', Fig. 4d), whereas the second input pattern ensured the required phase-shift (Fig. 4e). Interestingly, in spite of the difference in motor output, the network patterns had marginal visual differences in both behaviors (cf. Fig 4d-e, Supplementary Video 3). The neuronal phase-preference during the two behaviors were broadly scatter near the unity line (Fig. 4f), which indicates that many neurons kept their timing in the sequence. Roughly half of the neurons had a change in phase of less than $\pm 45^{\circ}$ (48.5\%) (Fig. $\mathbf{4 g}$-h). These observation were qualitatively similar to experimental observations, where the sequential activation, although not identical, remained during the two behaviors (Fig. 4i-m). We further tested whether other distinct motor patterns could be evoked in the BSG-model. A plethora of patterns or "gaits" could be induced via different activation profiles, with a diversity conspicuously similar to the diversity of real motor patterns (Extended Data Fig. 7-8). Generation of a desired motor pattern is a quest of finding the appropriate combination of neurons to modulate. We suggest this to be accomplished in trial-and-error-based motor learning ${ }^{31}$.

\section{Discussion}

In this report, we present compelling evidence that, rather than exhibiting push-pull activity, the spinal motor circuitry performs rotation. This is a low-dimensional dynamics, where the population continuously cycles through all phases and the radius of rotation correlates with the intended muscular force. The sequence involved in one behavior is similar to that of a different behavior (Fig. 4, Extended data figure 7-8) and the rotation even persisted in the enigmatic phenomenon of "deletions" (Extended data figure 9). These aspects of motor control have not previously been considered, and to explain these we had to develop a new theory, the 'Balanced Sequence Generator'. The hallmark is sequential activity in a balanced network, which is induced by tonic input from e.g. descending commands or sensory input. Such modulation of small selected subsets of neurons allowed generation of various motor programs with an unprecedented degree of diversity and flexibility. Interestingly, if the drive is brief or targeted, the model network could also generate non-rhythmic movement sequences, such as stroking a tennis ball or kicking a soccer ball. Hence, the BSG-theory could provide an important bridge between the motor circuits for rhythmic movement with those for non-rhythmic sequences, that has previously been absent. We predict such non-rhythmic movements to be generated by a single cycle of neural rotation, which is sculpted by selective gain modulation of the spinal network via descending commands from the brain or via sensory input.

\section{Acknowledgements (optional)}

Funding: This work was supported by The Independent research fund Denmark, and the Carlsberg foundation.

\section{Author contributions}

R.W.B. conceived the original experiments. P.C.P. setup and performed the experiments, collected the data and analyzed some of the data. M.W., H.L. and R.W.B. conceived the original theory. H.L. and R.W.B. designed, and developed the theory, analysed the experimental data, and wrote the manuscript.

\section{Competing interests}

The authors declare no competing interests.

\section{Supplementary Information}

All (other) data needed to evaluate the conclusions in the paper are present in the paper or the supplementary information. 

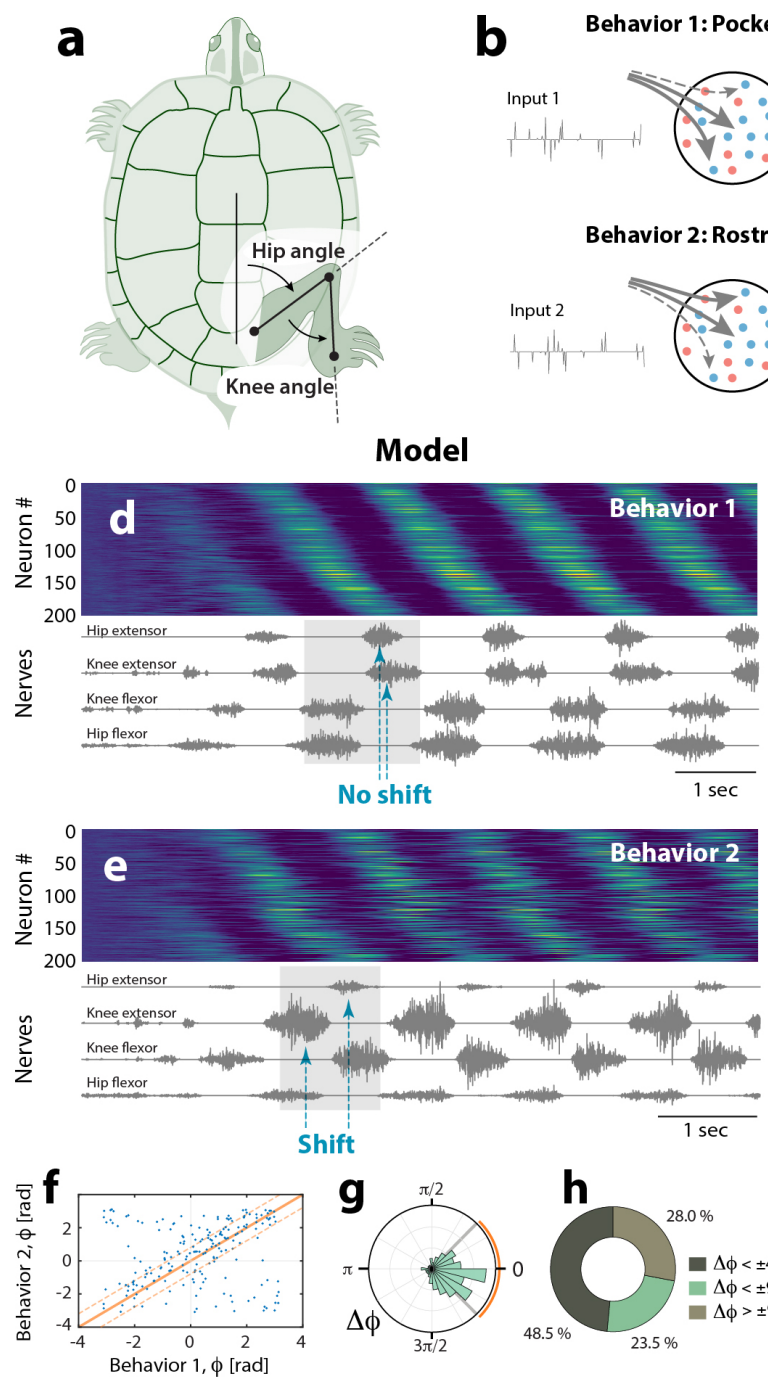
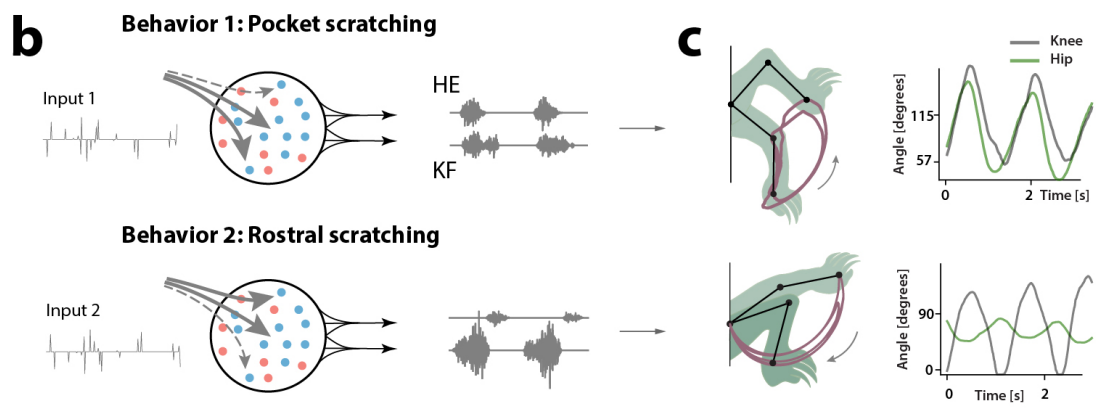

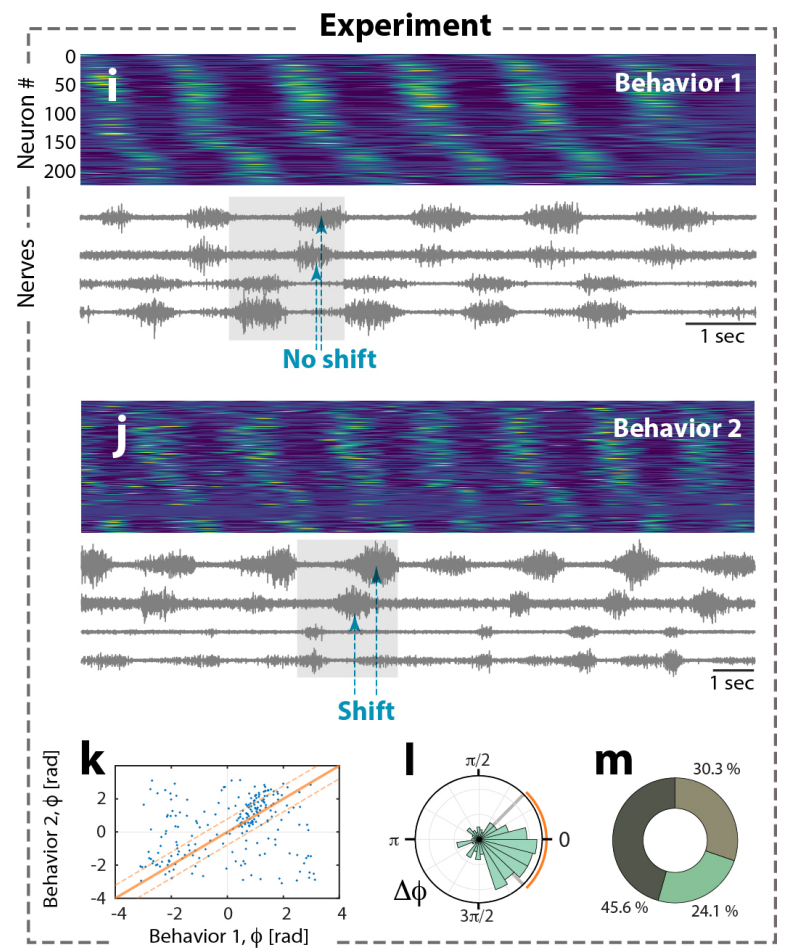

Fig. 4. $\mid$ Multifunctionalism in model and experiment. a, hindlimb movement is quantified using the hip and knee angles. b, two distinct motor behaviors (pocket- and rostral scratching) are evoked in BSG-model by distinct input profiles (left inset) of neuronal subsets. c, the evoked motor patterns (top: pocket, bottom: rostral) translated to limb trajectory (left, brown) and joint angles (right, hip and knee). d-e, associated ensemble activity are not identical, but have resemblance, although their motor patterns are qualitatively distinct ('no shift' vs. 'shift' in shaded region). f, phase $(\phi)$ of neurons in behavior $1 \mathrm{vs.}$ behavior 2 with respect to hip flexor scatter around the unity line, shown $\pm 45^{\circ}$. $\mathbf{g}$, phase difference $(\Delta \phi)$ in polar histogram. Orange line indicates $\pm 45^{\circ}$. $\mathbf{h}$, neuronal fraction with $\Delta \phi< \pm 45^{\circ}$ (dark), $\pm 90^{\circ}$ (green) and the rest (light brown). i-m, experimental results in similar arrangement as (d-h) and similar motor behaviors. (a) modified from ${ }^{30}$ with permission. 


\section{References}

1. Arber, S. \& Costa, R. M. Connecting neuronal circuits for movement. Science 360, DOI: 10.1126/science.aat5994 (2018).

2. Gosgnach, S. et al. Delineating the Diversity of Spinal Interneurons in Locomotor Circuits. J. Neurosci. 37, 10835-10841, DOI: 10.1523/jneurosci.1829-17.2017 (2017).

3. Goulding, M. Circuits controlling vertebrate locomotion: moving in a new direction. Nat Rev Neurosci 10, 507-518, DOI: $10.1038 / \mathrm{nrn} 2608$ (2009).

4. Grillner, S. \& El Manira, A. Current principles of motor control, with special reference to vertebrate locomotion. Physiol. Rev. 100, 271-320, DOI: 10.1152/physrev.00015.2019 (2020).

5. McCrea, D. A. \& Rybak, I. A. Organization of mammalian locomotor rhythm and pattern generation. Brain Res Rev 57, 134-146, DOI: 10.1016/j.brainresrev.2007.08.006 (2008).

6. Stein, P. S. G. \& Daniels-McQueen, S. Modular organization of turtle spinal interneurons during normal and deletion fictive rostral scratching. J. Neurosci. 22, 6800-6809, DOI: 20026654 (2002).

7. Petersen, P. C. \& Berg, R. W. Lognormal firing rate distribution reveals prominent fluctuation-driven regime in spinal motor networks. eLife 5, e18805, DOI: 10.7554/eLife.18805 (2016).

8. Radosevic, M. et al. Decoupling of timescales reveals sparse convergent CPG network in the adult spinal cord. Nat. Commun. 10, 2937, DOI: 10.1038/s41467-019-10822-9 (2019).

9. Churchland, M. M. et al. Neural population dynamics during reaching. Nature 487, 51-56, DOI: 10.1038/nature11129 (2012).

10. Sussillo, D., Churchland, M. M., Kaufman, M. T. \& Shenoy, K. V. A neural network that finds a naturalistic solution for the production of muscle activity. Nat. Neurosci. 18, 1025-1033, DOI: 10.1038/nn.4042 (2015).

11. Bruno, A. M., Frost, W. N. \& Humphries, M. D. A spiral attractor network drives locomotion in Aplysia. eLIFE 6, e27342, DOI: 10.7554/eLife.27342 (2017).

12. Berkinblit, M. B., Deliagina, T. G., Feldman, A. G., Gelfand, I. M. \& Orlovsky, G. N. Generation of scratching. I. Activity of spinal interneurons during scratching. J. neurophysiology 41, 1040-1057 (1978).

13. Auyong, N., Ollivier-lanvin, K. \& Lemay, M. A. Population spatiotemporal dynamics of spinal intermediate zone interneurons during air-stepping in adult spinal cats. J Neurophysiol 106, 1943-1953, DOI: 10.1152/jn.00258.2011. (2011).

14. Kwan, A. C., Dietz, S. B., Zhong, G., Harris-Warrick, R. M. \& Webb, W. W. Spatiotemporal dynamics of rhythmic spinal interneurons measured with two-photon calcium imaging and coherence analysis. J Neurophysiol 104, 3323-3333, DOI: 10.1152/jn.00679.2010 (2010).

15. Kiehn, O. et al. Excitatory components of the mammalian locomotor CPG. Brain Res Rev 57, 56-63, DOI: 10.1016/j. brainresrev.2007.07.002 (2008).

16. Rancic, V. \& Gosgnach, S. Recent insights into the rhythmogenic core of the locomotor cpg. Int. J. Mol. Sci. 22, 1-11, DOI: 10.3390/ijms22031394 (2021).

17. Hennequin, G., Agnes, E. J. \& Vogels, T. P. Inhibitory Plasticity: Balance, Control, and Codependence. Annu. Rev. Neurosci. 40, 557-79, DOI: 10.1146/annurev-neuro-072116-031005 (2017).

18. Berg, R. W., Willumsen, A. \& Lindén, H. When networks walk a fine line: balance of excitation and inhibition in spinal motor circuits. Curr. Opin. Physiol. 8, 76-83, DOI: 10.1016/j.cophys.2019.01.006 (2019).

19. Berg, R. W., Alaburda, A. \& Hounsgaard, J. Balanced inhibition and excitation drive spike activity in spinal half-centers. Science 315, DOI: 10.1126/science.1134960 (2007).

20. Machado, T. A. Probing circuits for spinal motor control. Ph.D. thesis, Columbia University Academic Commons (2015). DOI: doi.org/10.7916/D8TT4Q8J.

21. Ramirez, J.-M. \& Baertsch, N. A. The Dynamic Basis of Respiratory Rhythm Generation : One Breath at a Time. Annu. Rev. neuroscience 41, 475-99, DOI: 10.1146/annurev-neuro-080317-061756 (2018).

22. Rajan, K., Abbott, L. F. \& Sompolinsky, H. Stimulus-dependent suppression of chaos in recurrent neural networks. Phys. Rev. E - Stat. Nonlinear, Soft Matter Phys. 82, DOI: 10.1103/PhysRevE.82.011903 (2010).

23. Hennequin, G., Vogels, T. P. \& Gerstner, W. Optimal control of transient dynamics in balanced networks supports generation of complex movements. Neuron 82, 1394-1406, DOI: 10.1016/j.neuron.2014.04.045 (2014). 
24. van Vreeswijk, C. \& Sompolinsky, H. Chaos in neuronal networks with balanced excitatory and inhibitory activity. Science 274, 1724-6 (1996).

25. Beiran, M. \& Ostojic, S. Contrasting the effects of adaptation and synaptic filtering on the timescales of dynamics in recurrent networks. PLOS Comput. Biol. 15, DOI: 10.1371/journal.pcbi.1006893 (2019).

26. Stroud, J. P., Porter, M. A., Hennequin, G. \& Vogels, T. P. Motor primitives in space and time via targeted gain modulation in cortical networks. Nat. Neurosci. 21, 1774-1783, DOI: 10.1038/s41593-018-0276-0 (2018).

27. Gosgnach, S. et al. V1 spinal neurons regulate the speed of vertebrate locomotor outputs. Nature 440, 215-219, DOI: 10.1038/nature04545 (2006).

28. Callahan, R. A. et al. Spinal V2b neurons reveal a role for ipsilateral inhibition in speed control. eLife 8, 1-27, DOI: 10.7554/eLife.47837 (2019).

29. Berkowitz, A. Roles for multifunctional and specialized spinal interneurons during motor pattern generation in tadpoles, zebrafish larvae, and turtles. Front. Behav. Neurosci. 4, DOI: 10.3389/fnbeh.2010.00036 (2010).

30. Mortin, L. I., Keifer, J. \& Stein, P. S. Three forms of the scratch reflex in the spinal turtle: movement analyses. J. Neurophysiol. 53, 1501-1516 (1985).

31. Gallego, J. A., Perich, M. G., Miller, L. E. \& Solla, S. A. Neural Manifolds for the Control of Movement. Neuron 94, 978-984, DOI: 10.1016/j.neuron.2017.05.025 (2017). 